\title{
LOS INSTITUTOS ESCUELA EN CATALUNYA: RECUPERANDO LA MEJOR TRADICIÓN PEDAGÓGICA DE LA REPÚBLICA
}

\author{
INSTITUTOS ESCUELA IN CATALONIA: RECOVERING \\ THE BEST PEDAGOGICAL TRADITION OF THE REPUBLIC
}

http://dx.doi.org/10.15304/ie.28.5493

\author{
Màrius Martínez Muñoz \\ Universitat Autònoma de Barcelona \\ marius.martinez@uab.cat
}

Carmina Pinya Salomó

Profesora de enseñanza secundaria Departament d'Ensenyament (Generalitat de Catalunya)

cpina@xtec.cat

\section{RESUMEN}

El artículo ofrece la síntesis de un estudio desarrollado en 2011 en 11 de los 18 Institutos Escuela (IE) que funcionaban en Catalunya en el marco de la Ley de Educación de Catalunya. El estudio, financiado por el Consell Superior d'Avaluació del Sistema Educatiu y el Instituto de Ciencias de la Educación de la UAB, abordó la visión que de estas instituciones tenían los directivos de los centros, una muestra del profesorado, de los tutores y tutoras y de las familias, en los ámbitos organizativo, curricular, de orientación y de relación con el entorno. También se estudiaron dinámicas de coordinación entre centros de educación infantil y primaria y centros de secundaria en los mismos territorios con el fin de comprobar y contrastar otras dinámicas de coordinación en los mismos ámbitos en instituciones separadas.

Los resultados mostraron una visión muy positiva por parte de todos los colectivos vinculados a los IE y también permitieron identificar 50 prácticas de referencia en la coordinación de las dos etapas en los planos citados, que se reseñan también en el artículo.

El artículo termina con diez conclusiones que, en opinión de los autores, siguen perfectamente vigentes en la actualidad.

Palabras clave: Instituto Escuela; Calidad; Evaluación; Coordinación primaria secundaria; educación y territorio.

\section{ABSTRACT}

The article offers the synthesis of a study developed in 2011 in 11 of the 18 Institutos-Escuela (IE) that worked in Catalonia within the framework of the Education Law of Catalonia. The study, funded by the Consell Superior d'Avaluació del Sistema Educatiu and the Institute of Education Sciences of the $\mathrm{UAB}$, addressed the vision of these institutions had the directors of the centers, a sample of teachers, tutors and families, in the organizational, curricular, orientation and relationship with the environment. Coordination dynamics were also studied between early childhood and primary education centers and 
secondary schools in the same territories in order to verify and contrast other coordination dynamics in the same areas in separate institutions.

The results showed a very positive view on the part of all the groups linked to the IE and also allowed to identify 50 reference practices in the coordination of the two stages in the aforementioned planes, which are also reported in the article.

The article ends with ten conclusions that, in the opinion of the authors, are still perfectly valid today.

Keywords: Instituto Escuela; Quality; Evaluation; Secondary primary coordination; education and territory.

\section{INTRODUCCIÓN}

El 3 de febrero de 1932, tras la experiencia iniciada en Madrid en 1918, el Palacio del Gobernador del parque de la Ciutadella en Barcelona acogía por primera vez en Catalunya a sesenta estudiantes del Instituto Escuela de la Generalitat de Catalunya. Antes, en octubre de 1931, el ministro de Instrucción Pública y Bellas Artes había autorizado a la Generalitat a organizar en Barcelona "de acuerdo con las especiales necesidades y características de la región catalana, un instituto escuela de segunda enseñanza con carácter de ensayo pedagógico". Aquel mismo mes, el gobierno de la Generalitat promulgaba el decreto de creación del primer centro de enseñanza secundaria nacido al amparo de la autonomía política alcanzada en la época republicana. El funcionamiento del Instituto Escuela (IE) lo determinaban las normas elaboradas por el consejo de cultura de la Generalitat que fijaba, entre otras normas, la edad mínima de ingreso en el centro - 11 años- la admisión de alumnado de ambos sexos, el número de alumnos, el plan de enseñanza y la organización del centro. Estas normas se complementaban con un reglamento interno que definía los rasgos pedagógicos y organizativos de la institución: la completa coeducación, el uso del catalán como lengua básica, el laicismo en la teoría y las costumbres, la supresión de las calificaciones y los libros de texto -a favor de los libros, en su conjunto-, la preeminencia de la formación social y moral, la comprensión de la cultura, el civismo como ámbito global, las salidas fuera del aula, la introducción del ejercicio físico, la importancia de la música y la continuidad coordinada de la enseñanza primaria a la secundaria. La Ley de Educación de Catalunya, aprobada en el Parlament de Catalunya en julio de 2009, permitió de nuevo la creación de esta modalidad de establecimiento educativo que integra en un único centro, los niveles de educación infantil, primaria y secundaria obligatoria recuperando la mejor tradición pedagógica iniciada ochenta años antes. A finales de 2011, el Consell Superior d'Avaluació del Sistema Educatiu, encarga al ICE de la UAB una investigación evaluativa sobre los IE, con la propuesta de analizar once de las dieciocho instituciones que ya estaban en funcionamiento en Catalunya. Más allá del interés por los resultados educativos, interesaba especialmente identificar las prácticas diferenciales que esta tipología de centros desarrollaba, concretamente en los ámbitos de la organización escolar, la coordinación curricular y la orientación y tutoría del alumnado. Al mismo tiempo algunos de los rasgos de estos centros pueden tenerse en cuenta para impulsar medidas de optimización de la coordinación de centros de primaria y secundaria de una zona educativa que, aunque permanezcan separados, podrían mejorar su articulación en el territorio favoreciendo procesos y resultados educativos. Durante los posteriores años, el informe elaborado tras el estudio, del que se presenta una síntesis en este artículo, ha servido para impulsar nuevos centros de esta tipología con el convencimiento que prestan una inestimable labor al servicio de la educación de la ciudadanía. 


\section{UN MARCO REFERENCIAL PARA LOS INSTITUTOS ESCUELA: ESTRUCTURAS EDUCATIVAS, TRANSICIÓN Y ARTICULACIÓN ENTRE PRIMARIA Y SECUNDARIA}

\subsection{El marco normativo}

La ley 12/2009 de 10 de julio de Educación de Catalunya (LEC), aprobada por el Parlament de Catalunya permitió de nuevo la creación de la modalidad de los Institutos Escuela (IE) como establecimientos públicos de Educación en Catalunya. En concreto, el artículo 75 define los IE como aquellos centros educativos que imparten educación primaria y secundaria, entre otras enseñanzas de régimen general, permitiendo la integración en un solo centro de alumnos de los niveles de educación infantil, primaria y secundaria. En la misma ley se contemplaba la adscripción de centros educativos que pertenecen a la misma zona educativa, con la finalidad de ordenar el proceso de escolarización y de facilitar la continuidad educativa para todo aquel alumnado que siguiese cursando sus estudios en centros no integrados. Al mismo tiempo, también se puede determinar la adscripción educativa entre centros de una misma zona cuando éstos comparten los mismos objetivos del proyecto educativo. En el decreto 102 de 2010, que desarrolla la LEC y en las posteriores resoluciones de 16 de junio de 2011 se prevé la necesaria coordinación entre centros vinculados de educación primaria y secundaria a fin de garantizar una transición adecuada entre el alumnado de las dos etapas. Esta última cuestión lleva a regular la planificación, durante el curso escolar, de sesiones de coordinación que contribuyan a dar coherencia al proceso educativo y al itinerario formativo del alumnado. En estas sesiones se deben acordar los criterios de actuación comunes y compartidos, poniendo especial atención al conocimiento del alumnado que promociona, a la concreción y desarrollo del currículum, a los planteamientos del centro en relación a la inclusión y a los criterios de elaboración de las actividades de refuerzo y al seguimiento del proceso de adaptación en secundaria. Esta estructura de coordinación debe estar prevista y planificada. Al mismo tiempo se prevé una formación conjunta del profesorado en el marco de los planes de formación de zona.

\subsection{Las estructuras educativas en una perspectiva internacional}

De acuerdo a la clasificación internacional de la UNESCO (con seis niveles educativos delimitados en la International Standard Classification of Education o ISCED, que van de la educación pre primaria o ISCED 0 a la Educación de tercer ciclo superior o ISCED 6, destinada al doctorado, en la que ISCED 1 es la educación primaria y ISCED 2 la secundaria inferior, EURYDICE (2011) describe aquellos sistemas educativos en los que los niveles 1 y 2 de la enseñanza pública están integrados y aquellos en los que están separados:

- ISCED 1 e ISCED 2 integrados (estructura que correspondería a los Institutos Escuela): Bulgaria, Republica Checa, Dinamarca, Estonia, Hungría, Eslovenia, Eslovaquia, Finlandia, Suecia, Islandia, Noruega, Croacia y Turquía.

- ISCED 1 e ISCED 2 separados: Alemania, Bélgica, Irlanda, Grecia, España, Francia, Italia, Chipre, Lituania, Luxemburgo, Malta, Países Bajos, Austria, Polonia, Portugal, Rumanía, Reino Unido y Suiza. 
Esta primera aproximación debe complementarse con otros aspectos a considerar en el análisis de las estructuras: el papel de la administración educativa, la distribución de la titularidad de los centros (público / privado), los sistemas de gestión de la heterogeneidad y finalmente los sistemas de orientación y tutoría. A continuación, se examinan brevemente todos ellos:

\section{El papel de la administración educativa y la distribución de los centros (público / privado)}

El papel de las administraciones es distinto según los países. Así, se observa como en los países nórdicos existe un alto grado de descentralización y son los municipios y la administración supralocal los que gestionan la educación en el territorio más próximo. La administración central fija el marco curricular y las normas generales relativas a la calidad y la supervisión de la educación. En Alemania son las regiones las que disponen de autonomía para gestionar el servicio educativo y es la administración federal la que vela por el funcionamiento general del sistema y en Francia, por citar el ejemplo opuesto, la educación está altamente centralizada y es el estado central el que provee y regula la totalidad del sistema. Dichas diferencias inciden en la naturaleza de las decisiones que en materia educativa se toman, oscilando entre decisiones muy contextualizadas y cercanas a las características y necesidades de la ciudadanía a decisiones mucho más alejadas y de carácter general para el conjunto del sistema.

En relación a los sistemas públicos o privados de provisión de los servicios educativos, el debate es poliédrico, pero a los efectos de la estructura de los centros, en los países nórdicos donde la inmensa mayoría de los centros son públicos, el sistema es de no diferenciación de itinerarios en edades tempranas y de niveles de equidad semejantes entre centros. En Catalunya, la diferenciación entre centros de titularidad pública y centros de titularidad privada sigue generando diferencias de equidad en el sistema educativo. También se puede observar como un número muy importante de centros de titularidad privada concentran en el mismo establecimiento los niveles de educación infantil, primaria y secundaria mientras que el grueso de los centros públicos son de educación infantil y primaria por un lado y de educación secundaria por otro. En Suecia, se pudo comprobar como el incremento ligero de los centros privados -y su consiguiente diferenciación- que alcanzó el 10\% del total, produjo un incremento asociado de las desigualdades y un descenso de la equidad del sistema (EURYDICE, 2011g).

\section{Los sistemas de gestión de la heterogeneidad}

Uno de los debates sobre las estructuras educativas en el contexto internacional tiene que ver con la gestión de la heterogeneidad del alumnado en los itinerarios obligatorios. Una primera aproximación permite diferenciar los sistemas comprensivos de los sistemas selectivos o diferenciados. Una mirada más analítica permite vislumbrar la complejidad de dicha cuestión debido, entre otras cosas, a la aparente contradicción o tensión dentro de los mismos sistemas que han de promover la integración y la cohesión sociales y a la vez diferencian y jerarquizan en función de los requisitos del mercado de trabajo, de las capacidades o de los resultados académicos. A partir del siglo XX y una vez garantizada la formación básica o primaria, el debate sobre la diferenciación y los itinerarios formativos se traslada a la educación secundaria inferior que, una vez también 
generalizada, traslada el debate al momento en el que se deben diferenciar los itinerarios académicos de los profesionalizadores, por ejemplo.

Una primera aproximación a la cuestión permite identificar cuatro grandes estrategias: la creación de itinerarios formativos diferenciados, la utilización de agrupaciones homogéneas por habilidad, la repetición de nivel y las estrategias de individualización y tutoría.

En Alemania y Austria, por ejemplo, existen itinerarios o vías específicas introducidas a los 10 o 11 años mientras que, en los países nórdicos, el currículum común continúa hasta los 16 años. Los sistemas no comprensivos tienen una tasa de desgaste, en términos de repetición y abandono, en contraste con los sistemas comprensivos que no utilizan la diferenciación organizativa o prácticas de agrupación. Los sistemas comprensivos suelen tener un impacto positivo especialmente en términos de equidad. Las formas de diferenciación o las divisiones entre centros educativos tienden a incrementar las desigualdades sociales en el rendimiento. En los países con cultura de la diferenciación, los resultados del alumnado más vulnerable no mejoran e incluso se identifica una relación negativa entre el índice de diferenciación y los resultados o puntuaciones medias en algunas pruebas como las de lectura.

Tal como señalan Dupriez, Dumay y Vause (2008), los estudios internacionales demuestran que aquellos sistemas basados en itinerarios tempranos se caracterizan por una fuerte relación entre los resultados del alumnado y el contexto cultural. Estos autores estudian las estrategias utilizadas con el alumnado más vulnerable e identifican tres estrategias: las rutas académicas, la utilización de ambientes educativos y la utilización de los niveles de logro como criterio. Estos mismos autores identifican cuatro modelos de ajuste: el modelo de separación (Alemania, Austria, Hungría): el modelo de integración uniforme (España, Portugal, Francia), el modelo de integración a la carta (Estados Unidos) y el modelo de integración individualizado (Dinamarca, Islandia, Finlandia, Noruega y Suecia). En este último no hay apenas repetición de curso ni diferenciación por habilidad ni itinerarios sino más bien un proceso de individualización en el aula, una tutorización individualizada y un trabajo en pequeños grupos para que todo el alumnado domine el currículum a un ritmo similar.

\section{La orientación y tutoría del alumnado}

La Orientación y la tutoría son procesos a tener en cuenta, tanto si el alumnado cursa sus estudios en un sistema integrado, como si lo hace en uno diferenciado. Si bien todos los sistemas disponen de servicios, acciones y programas de orientación, no en todos empieza en el mismo momento ni se desarrolla del mismo modo. Así, en Francia, por ejemplo, la orientación propiamente dicha se inicia en la enseñanza secundaria y en España, la orientación en primaria tiene que ver más con los aspectos escolares, de carácter académico y personal y se materializa fundamentalmente en la tutoría. En secundaria se amplía con la orientación profesional que suele intensificarse al final de la ESO a través de acciones esencialmente puntuales. En general, no es hasta la educación secundaria que la orientación se generaliza a través de actividades de orientación individual y grupal siendo el tutor o tutora quien desarrolla el grueso de la actividad orientadora. La orientación se concreta también en los procesos de transición entre la primaria y la secundaria que nuevamente suelen ser puntuales y concentrados en el momento temporal en que se materializa dicha transición 
(final de sexto de primaria y muy, muy al principio de secundaria). Si bien la orientación se concreta en acciones dirigidas al desarrollo personal, académico y vocacional - profesional del alumnado, existen particularidades en relación al énfasis o a los aspectos que se incluyen bajo estas denominaciones genéricas. Así, por ejemplo, en Noruega, se considera que la orientación ha de prevenir el abandono, reducir las diferencias sociales e integrar a las minorías étnicas a partir de la adopción de una perspectiva holística del alumnado y de una visión articulada de la orientación personal y social con la educativa - académica- y vocacional. En Finlandia añaden la importancia de orientar al alumnado para ejercer una ciudadanía activa, enfatizando la importancia de fijar la atención en los momentos de transición para obtener los mejores resultados para todo el alumnado. Es evidente que muchos de estos conceptos están presentes en los ordenamientos de los distintos sistemas educativos, pero es sólo en algunos donde se vinculan con el proceso de desarrollo y transición entre etapas, aspecto importante al hablar de los Institutos Escuela desde la óptica de la relación y articulación entre primaria y secundaria.

\subsection{Los conceptos de traspaso y transición y sus desafíos}

Las relaciones entre las etapas de enseñanza primaria y secundaria se pueden considerar desde múltiples enfoques, más allá de constituir estructuras integradas o diferenciadas. Para analizar dichas relaciones, de acuerdo con la bibliografía internacional consultada, se consideran, por su interés, los conceptos de traspaso y transición (transfer y transition en su acepción original en inglés), las necesidades y desafíos que presentan y las estrategias que se desarrollan para afrontarlas.

Tal y como apuntan Galton, Morrison y Pell (2000), a menudo los conceptos de transfer y transition 1 se usan como intercambiables refiriéndose al paso de la primaria a la secundaria (lo cual implica, en algunos sistemas, un cambio de escuela o institución). Un examen más detallado permitiría defender un uso diferenciado de ambos términos: transición, para hacer referencia al cambio de escuela o institución en el paso de primaria a secundaria (como ocurre en el grueso del sistema público en Catalunya y en el resto de España), que afecta a todo el grupo que debe moverse de un centro a otro, con todos los desafíos que ello implica. Traspaso, para designar el paso o cambio de nivel que cada año hace el alumnado para ir progresando en la etapa, dentro de la misma institución o establecimiento. Así, un alumno que cursa su enseñanza en un centro de titularidad pública experimenta procesos de traspaso cada año y generalmente, excepto en los Institutos Escuela, una transición al pasar de primaria a secundaria. Esta diferenciación permite, por un lado, recuperar los procesos de cambio en los diferentes niveles o cursos que tendrán sentido al hablar de articulación entre la educación primaria y secundaria, y considerar el proceso gradual de transformación en las maneras de trabajar, en la aproximación a los contenidos, en el proceso de creciente autonomía y en otros aspectos, en una perspectiva diacrónica mucho más completa.

En la literatura anglosajona manejada se utiliza el concepto de transfer para referirse al cambio de institución y el de transition para considerar el cambio de nivel. En Catalunya y el contexto español, no hay tradición en el uso de dichas acepciones por lo que la traducción a nuestro contexto ha mantenido el término transición para referirse al cambio de etapa y el transfer se ha traducido como traspaso para designar el cambio de nivel. 
Además, en el caso de los IE, al analizar el cambio de primaria a secundaria, la perspectiva del traspaso ayuda a entender mejor cómo debería entenderse dicho proceso, acercándose más al concepto de traspaso que no a una transición completa al tratarse de una misma institución. Sin embargo, el concepto de transición se mantiene porque en el sistema público, con la excepción de los IE, como ya se ha mencionado, se mantienen las etapas primaria y secundaria en instituciones separadas. También es relevante porqué el cambio de etapa, aún en una misma institución, supone cambiar lógicas curriculares y de interacción docente-discente que no deben pasarse por alto.

Todos estos procesos se han abordado tradicionalmente desde una lógica evolutiva (considerando factores psicobiológicos) si bien en las dos últimas décadas ha emergido una visión sistémica que considera los factores externos a la persona de carácter contextual e institucional (Anderson, Jacobs, Schramm y Splitgerber, 2000: 326). En este estudio se ha considerado la visión sistémica asumiendo la enorme importancia que sigue teniendo la dimensión evolutiva.

Los desafíos de los procesos de traspaso y transición son múltiples, tal y como lo ponen de manifiesto diversos estudios (Gradaïlla, Querol y Bundó, 2011; Ruiz Guevara y otros, 2010, Anderson et al., 2000, Galton et al., 2000). La tendencia a la disminución de la seguridad personal frente a ambientes nuevos con nuevas y crecientes exigencias, los cambios en los patrones, culturas y las relaciones sociales y los cambios de carácter organizativo, metodológico, comunicativo, de registro, de distribución del tiempo (horarios y calendarios), el incremento del tiempo de estudio y su complejidad, entre otros, pueden identificarse con facilidad (Martínez y Pinya, 2012).

Ya en 1996 Gimeno Sacristán señalaba que el 45\% del alumnado manifestaba dificultades de adaptación a la nueva etapa que iban acompañadas de un descenso en el rendimiento escolar. Según Galton et al. (2000) el 40\% del alumnado experimenta un hiato - una rotura o salto - en el proceso de cambio entre primaria y secundaria atribuido en muchas ocasiones a la falta de continuidad del currículo pero también a las variaciones en la forma de enseñar, cuestiones que se agravan en aquellos alumnos con especiales dificultades para el cambio que pueden presentar o haber presentado: problemas previos de comportamiento, poca preparación académica, poca madurez o estrategias para afrontar nuevas situaciones, falta de autoconfianza y autoestima, contextos socioeconómicos desfavorecidos, falta de apoyo familiar durante la escolaridad y especialmente en momentos de cambio. Este alumnado experimenta problemas de rendimiento, como ya se ha mencionado, suele tener más dificultades en la relación con el profesorado y con el resto de pares y, en definitiva, mayores dificultades de adaptación al nuevo contexto tanto desde el punto de vista físico, como social y académico (Galton et al., 2000; Galton y Morrison, 2000). Todos estos cambios y dificultades pueden ser consideradas discontinuidades que tienen una vertiente social y otra organizativa, pero que no tienen que suponer un problema o un declive permanente en el rendimiento y en el proceso de desarrollo global pero sí deben constituir un foco de atención prioritaria para que no se conviertan en un problema real de continuidad si no se afrontan y gestionan adecuadamente desde las instancias organizativas, curriculares y de orientación.

Los desafíos señalados constituyen un conjunto de necesidades y problemáticas de articulación entre primaria y secundaria que deben afrontar los centros educativos que trabajan en un mismo territorio. En algunos casos el primer problema, desde esta lógica territorial, es justamente la falta de coordinación y la falta de información, que es la base para la toma de decisiones, y que no 
siempre fluye o se tiene en cuenta en momentos de transición. A menudo, cuando se usa información para el traspaso o la transición, se hace más caso a la información de carácter administrativo que a la que tiene contenido pedagógico y de orientación. El desconocimiento de los proyectos educativos de las instituciones por parte de la comunidad, pero en ocasiones también por parte del propio profesorado genera un riesgo de desvinculación con el mismo de efectos diversos no deseados. Se deben considerar también la discontinuidad metodológica y curricular que, aunque la legislación puede intentar articular, a menudo, las características diferenciales entre profesorado de una y otra etapa y la existencia de culturas profesionales e institucionales distintas la dificultan provocando un riesgo de desafección que, a su vez, unido a las dificultades ya mencionadas, acerca al alumno al riesgo de abandono o fracaso, expresión final de una combinación de factores organizativos, curriculares, de orientación, personales, sociales y emocionales.

Dos factores que deben añadirse a la ecuación son el papel de las familias y su relación con el proceso y las instituciones educativas (también las acciones de las instituciones para facilitar dicha relación) y las cuestiones relacionadas con la equidad y por lo tanto con el trabajo específico en los contextos más vulnerables socioeconómicamente.

\subsection{Estrategias para optimizar el proceso de transición y el cambio}

Tal y como destacaban Galton y Morrison (2000), los procesos de optimización de los cambios y transiciones han tenido más que ver con la eficiencia administrativa que con las cuestiones personales y sociales del alumnado. Para abordar dicha optimización desde esta segunda perspectiva, se debe poner el énfasis en los aspectos de carácter pedagógico. Por ello, siguiendo el hilo del apartado anterior, parece imprescindible propiciar la coordinación territorial a partir de considerar zonas de influencia en las que los centros de primaria y secundaria colaboran en estas tareas como conjunto estable de centros. En este contexto es más fácil articular procesos, optimizar la coordinación y fluidificar las dinámicas que se pierden si no existe este criterio de territorio o de acción de proximidad. En este mismo contexto, disponer de información fiable y precisa sobre las etapas es también importante, tanto si se trata de establecimientos diferenciados como si se trata de un mismo establecimiento. Otro elemento que debe considerarse, en relación con los procesos de articulación e integración de las dinámicas educativas, tiene que ver con la identidad de los centros (Pietarinen, 2000; Hargreaves, 1994): para articular etapas y buscar lógicas de coordinación y acompañamiento, se deben producir cambios en las culturas y los climas escolares que guardan una relación directa con sus respectivas identidades, proyectos y carácter. Por ello es importante disponer de un proyecto y de una identidad actualizados y conocidos y también de un sentimiento de pertenencia que se genera alrededor de las prácticas y los valores compartidos, así como de los esfuerzos para que sean conocidos y valorados por parte de toda la comunidad. Además, la pertenencia se relaciona dialécticamente con la participación. Las transiciones y traspasos se optimizan cuando hay equipos estables que se sienten parte de un proyecto curricular, organizativo, de orientación y acompañamiento, en el que también participan activamente las familias.

La coordinación curricular implica no partir de cero en secundaria sino tener presente la etapa anterior y conectarla con la etapa en curso, dando sentido y continuidad vertical a las diferentes áreas del currículo y horizontal, en la propuesta curricular de cada nivel. 
La coordinación metodológica implica también una cierta continuidad en las propuestas estratégicas y de actividades, en un proceso gradual de creciente participación, autonomía y autogestión. La coordinación ha de contemplar también los recursos y la evaluación.

En relación a la orientación, se debe tener en cuenta que los cambios de etapa pivotan sobre el progreso académico y educativo y por ello se debe proporcionar un apoyo específico al estudio y a las estrategias de aprendizaje, un esfuerzo por incrementar la preparación académica pero también las estrategias para afrontar los cambios y, en definitiva, en la competencia como estudiante que puede concretarse en el conjunto de competencias de autonomía e iniciativa personal, de aprender a aprender, la social y ciudadana. Este esfuerzo orientador es también coral y debe contar con la participación del profesorado, tutores y tutoras, los iguales y la familia, además de otros agentes del territorio que pueden también participar en algún momento del proceso.

Anderson et al. (2000), partiendo de otras investigaciones, destacan cinco componentes o acciones esenciales para un modelo de transición sistémica como el que ocupa este artículo:

1. Desarrollar un equipo coordinador y planificador que incluya diferentes agentes y responsables del centro.

2. Generar objetivos e identificar problemas.

3. Desarrollar un plan escrito de transición y cambio que contemple las distintas necesidades y las distintas actividades en una perspectiva temporal larga (transición) y multifacética (personal, social, académica y organizativa).

4. Conseguir el apoyo y compromiso del profesorado y de todos aquellos profesionales vinculados con el proceso de transición.

5. Evaluar la transición.

\section{BREVE RESEÑA DE LA METODOLOGÍA DEL ESTUDIO}

El estudio que se desarrolló en Catalunya por encargo del Consell Superior d'Avaluació del Sistema Educatiu, se llevó a cabo el curso académico 2011/2012, en 11 de los 18 IE de que disponía Catalunya en aquel momento. Se descartaron 2 centros por tener una antigüedad menor a 2 años en su funcionamiento. También se estudiaron las relaciones entre primaria y secundaria de otros tantos centros de características similares y ubicación geográfica análoga a los IE seleccionados, con el fin de comprobar las estrategias de coordinación curricular, organizativas, de orientación y de relación con el entorno.

El trabajo de campo contempló la administración de cuestionarios a una muestra de equipos directivos, orientadores, tutores y familias. Los cuestionarios preguntaban sobre la percepción / valoración que los distintos encuestados tenían en relación a los aspectos de coordinación curricular, organizativa, de orientación y de relaciones con el entorno. Las preguntas tenían dos versiones: una que preguntaba sobre la percepción acerca de la situación actual del centro y otra que preguntaba sobre la situación que, en su opinión, idealmente debería tener. El objetivo era identificar las percepciones sobre la situación del centro, pero también identificar aquellos ámbitos en los que había 
mayores expectativas o aspiraciones y también mayores discrepancias entre la situación actual y la deseada o deseable. Las respuestas también deberían permitir contrastar la visión que los distintos colectivos encuestados tenían sobre las distintas dimensiones consideradas. También, las respuestas de los colectivos pertenecientes a los IE podían contrastarse con las de los colectivos pertenecientes a los establecimientos segregados.

Además de los cuestionarios, se realizaron 8 visitas para desarrollar entrevistas con responsables de cuatro IE, dos Institutos de secundaria y dos escuelas de primaria, con el objetivo de identificar prácticas de referencia relacionadas con la coordinación, las transiciones y los traspasos, en sus vertientes curriculares, organizativas y de orientación.

La descripción detallada de la metodología se puede consultar en el informe que publicó la Generalitat de Catalunya en 2012, en la colección Documents, número 21 (Martínez y Pinya, 2012).

\section{RESULTADOS}

Una primera aproximación a los resultados mostró como las percepciones más positivas en todas las dimensiones fueron mostradas por los encuestados de los IE y las más críticas las de los institutos de secundaria, si bien el profesorado más crítico fue el de primaria y las familias más críticas las de secundaria, en relación a las cuestiones planteadas. Los cargos directivos presentaron las valoraciones más positivas en el caso de los IE y los de los Institutos las menos optimistas. Los docentes con cargos de coordinación son los más críticos tanto en instituciones separadas como en los IE.

En la vertiente cualitativa, el profesorado de los IE considera como valores centrales de sus centros la coherencia en la línea pedagógica en las etapas obligatorias de enseñanza. También valoran la importancia de dicha coherencia en la promoción del alumnado, el enraizamiento en el entorno y el conocimiento mutuo y la participación de la comunidad educativa en el centro. Al mismo tiempo las familias de los IE valoran la educación integral del alumnado en una perspectiva continua.

En las escuelas, se identificaron, como valores centrales el respeto a todas las personas, la apertura a la participación de toda la comunidad para la compensación de desigualdades y el fomento del desarrollo integral del alumno. También el trabajo cooperativo de los docentes y la potenciación de cada alumno como sujeto en el centro de su proyecto de aprendizaje. Las familias de las escuelas destacan el respeto a la diversidad y la inclusión de todo el alumnado, así como el fomento de la igualdad de oportunidades.

En los institutos el valor central mostrado es el de la integración de todo el alumnado, el fomento de la cohesión social y el respeto a la interculturalidad. Destacan como actividades representativas de su funcionamiento, los intercambios internacionales y las actividades de carácter cultural. Las familias, por el contrario, identifican poco los acontecimientos que muestran el talante del centro y sus respuestas son mucho más heterogéneas.

Los aspectos de orientación siguen recayendo, en la percepción colectiva, en los tutores y tutoras con el apoyo en algunos casos de las coordinaciones y las personas responsables de la 
orientación. En pocos casos se hace referencia al papel de todo el equipo docente en las cuestiones relacionadas con la transición o los traspasos.

El trabajo permitió identificar 50 prácticas de referencia que se presentan de manera muy esquemática y que permitieron constatar que la coordinación organizativa, metodológica, curricular, de evaluación y de apoyo a las transiciones y traspasos se materializan de manera diversa tanto en los IE (espacio privilegiado para ello por tratarse de un mismo establecimiento) como también en algunos territorios en los que los distintos centros educativos han ido consolidando redes de colaboración a distintos niveles.

A continuación, se reseñan algunas de ellas, agrupadas en 6 categorías:

1. Prácticas de coordinación territorial

2. Prácticas de conocimiento del proyecto pedagógico del centro

3. Prácticas de vinculación al centro

4. Prácticas para la continuidad en los currículos y en las metodologías

5. Prácticas de orientación, tutoría y apoyo emocional

6. Prácticas de implicación de las familias

\section{Prácticas de coordinación territorial}

Esta coordinación fomenta la articulación de las propuestas pedagógicas de los centros de un mismo territorio. En un sistema de titularidad pública, en el que pocos centros tienen integradas las etapas de primaria y secundaria, merece la pena reconocer las prácticas que fomentan la cultura de la colaboración entre centros. Estos proyectos ayudan a compensar desigualdades y a buscar itinerarios más favorables para aquellos colectivos más vulnerables. Recuperan la idea de zona educativa y de acción colaborativa, coordinada y de proximidad:

- Coordinación de centros de zona y seminarios de coordinación entre primaria y secundaria: a cargo de las personas responsables de las jefaturas de estudio y de las coordinaciones pedagógicas que gestionan el traspaso de información y las actividades que permitirán hacer más fluida la transición. También los Institutos y las escuelas que tienen adscritas funcionan coordinadamente en los planos curriculares, pero también de seguimiento y retroalimentación de los distintos niveles, en función del progreso de los alumnos. En estos procesos pueden intervenir también los servicios educativos del territorio (Centros de recursos pedagógicos, Equipos de asesoramiento psicopedagógico) y la inspección educativa.

- Actividades conjuntas de alumnado de sexto de una zona educativa: salidas en las que alumnos de distintos centros, que luego se encontrarán en la ESO, comparten propuestas educativas, lúdicas, o culturales antes de su llegada al instituto. Buscan justamente que el alumnado de distintos centros se mezcle en la actividad propuesta.

- Redes 0-18: Nacen para dar respuesta a las necesidades de coordinación entre centros con alumnado entre 0 y 18 años. Generan actividades compartidas entre el alumnado de distintos centros, y seminarios de coordinación, de formación u otras actividades que se organizan con el apoyo de los centros de recursos pedagógicos. 
- Planes educativos de entorno: en los que se organizan actividades escolares y extraescolares que crean red en la comunidad y compensan desigualdades.

\section{Prácticas de conocimiento del proyecto pedagógico del centro}

Pretenden promover el conocimiento de las propuestas pedagógicas por parte del alumnado, de sus familias y también del profesorado de ciclo superior de primaria.

- Padrinos de transición: Alumnos de primero de ESO, seleccionados a tal efecto, apadrinan a grupos de alumnos de primaria. En reuniones previas reciben formación para desempeñar tal tarea. Una vez cursen primero, también tienen la misión de hacerles un seguimiento.

- “Paso a la ESO: ¡No pasa nada!”: Es un proyecto telemático de comunicación entre alumnado de sexto y alumnado de primero. Se complementa con reuniones presenciales donde se "reencuentran" con las personas con las que han estado comunicándose para resolver dudas o temores sobre la ESO.

- "Encuentro de El paso": Con la tutorización de alumnado de bachillerato, alumnado de sexto y de primero realiza talleres y actividades diversas, en un centro o en una zona educativa. El profesorado especialista organiza los talleres, pero el alumnado los dinamiza.

- Visita al instituto: Que se concreta en una jornada de visita por parte del alumnado de sexto a su futuro instituto pero que supone un contacto y una preparación previa de los tutores de ambos niveles más el protagonismo del alumnado de primero de ESO que conduce la visita y recibe al alumnado visitante.

- Carpetas de grupo: El traspaso de información suele tener carácter individual e individualizado. Sin perder esta parte, las carpetas de grupo permiten traspasar información de las dinámicas grupales en primaria, de las actividades y proyectos más significativos, sobre todo en casos en los que un número muy elevado de alumnos irá a los mismos centros de secundaria o, si es un IE, seguirá compartiendo aula con muchos de sus antiguos compañeros.

\section{Prácticas de vinculación al centro}

Se integran un conjunto de actividades que promueven la mayor implicación del alumnado como también algunas actividades para familias o docentes.

- Teatro en inglés juegos de patio, dinamización de fiestas: Alumnado de ESO prepara historias cortas en inglés y las representa al alumnado de educación infantil y ciclo inicial del propio centro (IE) o de centros adscritos del territorio. En los juegos de patio, el alumnado de quinto organiza actividades de juego en el patio para el alumnado de educación infantil con una determinada periodicidad. En el caso de las fiestas, el alumnado de ESO se responsabiliza de dinamizar algunas de las fiestas tradicionales del centro como el carnaval. 
- Expliquemos cuentos, padrinos de lectura y de escritura: Alumnado de sexto lee con alumnos de P5 y alumnado de ESO ayuda al de primaria en la redacción de textos de distintos tipos. También el alumnado de sexto y de la ESO explica cuentos al alumnado de parvulario. Además del trabajo lingüístico, se promueven las relaciones internivel y el desarrollo de las competencias comunicativas.

- Visita a exposiciones, jornadas de ciencia: Alumnado de un nivel visita la exposición de trabajos o actividades de otro nivel, permitiendo cultivar el respeto, la curiosidad, pero también la familiarización con el tipo de trabajo y el entorno en el que desarrollarán su actividad cuando sean más mayores. Una variante son las actividades en el laboratorio preparadas por alumnado de ESO para alumnado de primaria.

- Proyectos y talleres internivel: El alumnado de los distintos niveles de educación infantil realiza proyectos conjuntos en grupos heterogéneos. Al final de la etapa, ha compartido actividades con alumnado de dos niveles por debajo y dos por encima del suyo. Esta experiencia y los vínculos internivel suavizan los traspasos y las transiciones. Participar en proyectos de redacción del web y de la revista escolar, en equipos de distintos niveles educativos sería también un ejemplo de estas dinámicas de trabajo internivel.

- Corresponsabilidad de los docentes del claustro: Se fomenta, a través del trabajo, de las reuniones, de las consignas y del proyecto, que todo el profesorado tenga responsabilidades más allá de la docencia y de los propios grupos de nivel. La idea que un docente de infantil tiene también responsabilidad educativa con cualquier otro alumno de primaria o de ESO mejora cualquier proceso educativo.

- Entrevista de la dirección con cada docente e información periódica al claustro y a las familias: Las entrevistas personalizadas de la dirección con cada docente permiten tener mejor conocimiento de las inquietudes, dinámicas y propuestas de todos. Informar regularmente (semanalmente o mensualmente), mediante comunicación electrónica, de lo más significativo que se está haciendo en el centro, también mejora la comunicación y el sentimiento de pertenencia.

- Participación de familias en comisiones y en el AMPA: Se promueven comisiones de trabajo en las que participan colaborativamente docentes y familiares para trabajar temáticas de interés compartido.

\section{Prácticas para la continuidad en los currículos y en las metodologías}

La continuidad curricular es quizás uno de los aspectos que presenta mayor dificultad de coordinación en la práctica por diversos motivos (falta de tradición, perfiles docentes muy distintos, falta de tiempo y espacios), sin embargo, es de los más importantes. Algunas prácticas se desarrollan entre centros y otras en el seno de los IE. Aunque se trate de una misma institución, las dificultades fueron igualmente señaladas.

- Propuesta coordinada de tareas de sexto curso: los docentes de sexto de una zona se coordinan para las tareas de verano con su instituto de referencia. La coordinación en el tipo de tareas, así como con los criterios de corrección y de evaluación formativa facilita 
el seguimiento y evaluación del trabajo del alumnado y permite el conocimiento mutuo entre centros.

- Trabajo internivel dentro el centro: Coordinación curricular, metodológica y de criterios de evaluación entre docentes de un mismo centro y también en el contexto territorial con la creación, por ejemplo, de grupos de trabajo con especialistas de la zona educativa.

- Horarios de reuniones comunes de todos los docentes: facilitan las oportunidades efectivas de coordinación horizontal y vertical y de trabajo conjunto entre docentes. Se promueven momentos de encuentro pedagógico en los que se tratan, de manera monográfica, aspectos de coordinación. Los claustros pedagógicos podrían ser una modalidad que lo ejemplificaría.

- Docencia y coordinación por ámbitos en secundaria: Se agrupan docentes por ámbitos que se coordinan vertical y horizontalmente. La docencia por ámbitos en secundaria permite además la interdisciplinariedad y evita la dispersión y la atomización de docentes en un grupo clase.

- Docencia en ambas etapas: Se procura que docentes de secundaria impartan docencia en primaria y al revés. La interacción entre las dos etapas y la superación de la línea divisoria aporta una mayor comprensión del desarrollo continuo del aprendizaje y mejora la relación con el alumnado y entre el profesorado.

- Retorno de las primeras evaluaciones a la escuela: Tras la primera evaluación de primero de ESO, se informa a los tutores de sexto de primaria de los resultados académicos y de la transición de los exalumnos. Esta práctica está muy bien valorada por los docentes de primaria que a menudo dejan de tener información sobre la evolución de sus exalumnos y sobre el éxito de la transición.

\section{Prácticas de orientación, tutoría y apoyo emocional}

Si bien el ámbito de la orientación no fue el que presentó más preocupación por parte de los encuestados, el número de prácticas identificadas fue especialmente reducido. Predominan las actividades de carácter informativo puesto que la coordinación sobre el currículo, a menudo no se asocia a la orientación. El énfasis se sitúa en prevenir la desafección y en el apoyo emocional.

- Adaptación del primer día de curso en ESO: el primer curso empieza un día antes que el resto de cursos con el fin de familiarizarse y apropiarse del espacio y no tener interferencias el primer día.

- Salida conjunta sexto / primero: En el marco de un proyecto interdisciplinar, se realiza una salida de uno o dos días con alumnado de sexto y de primero con un programa que enfatiza también los elementos de convivencia, dinámica grupal y conocimiento mutuo.

- Redistribución de la composición de los grupos en quinto (IE): En algunos Institutos Escuela se procede a reconfigurar los grupos clase en quinto, con el fin que el cambio de compañeros no se produzca al mismo tiempo que el cambio de etapa y para ajustar las composiciones atendiendo a dinámicas interpersonales. 
- Asambleas semanales de preparación de la transición: Durante algunos meses del sexto curso se realizan dos asambleas semanales para tratar aspectos relacionados con la transición. En algunos institutos se mantiene la actividad durante algunos meses tras la llegada de los nuevos alumnos en primer curso. Compartir con los compañeros las dudas y las vivencias facilita la transición.

- Co-tutorización o tutorización compartida: Se asignan dos tutores a un mismo grupo de alumnos en primero de la ESO para hacer un mejor seguimiento de sus trayectorias. Se buscan tutores veteranos que puedan dedicar más tiempo a cada alumno tutorizado. En algunos centros se llegan a asignar tutores para cada cinco alumnos con el fin de aproximar todavía más el seguimiento, incrementar el vínculo y evitar la desafección y el abandono.

\section{Prácticas de implicación de las familias}

La implicación de las familias se valora muy positivamente, pero a la vez, muchos centros reportan pocas actividades para fomentarla. Si bien las prácticas identificadas son de carácter esencialmente informativo, son un primer paso para su implicación.

- Puertas abiertas: Se invita a las familias a conocer las instalaciones y el proyecto de centro. Es quizás, la práctica más extendida y permite que las familias compartan las primeras experiencias relacionadas con la transición.

- Sesiones informativas sobre la ESO, su proyecto, sus dinámicas y metodologías: En la escuela: Las escuelas de primaria invitan a las familias a charlas sobre la ESO, a cargo de docentes, de los profesionales de los equipos de asesoramiento psicopedagógico o de conferenciantes externos. Permiten conocer los cambios que experimenta un alumno en el proceso de transitar a la nueva etapa. También se organizan charlas de este tipo en los institutos con una presentación más detallada de aspectos operativos (horarios, calendarios, actividades, normativa, proyecto pedagógico).

- Sesiones formativas: En el marco de un café o una merienda, se invita a las familias a charlas o seminarios formativos sobre temáticas específicas de interés educativo. En ellas se generan vínculos de las familias con el centro y también se abordan de manera más informada, determinadas cuestiones de interés.

- Entrevistas de tutoría para preparar la transición y la acogida en primero de ESO: Las entrevistas con las personas responsables de la tutoría, tanto en sexto como en primero, permiten un trabajo individualizado de la transición, con el protagonismo del alumno y la participación de las familias lo cual permite un mejor acompañamiento del proceso durante dos años.

- Reunión del primer día de curso en el instituto: La primera tarde del nuevo curso en la ESO se convoca a las familias a una reunión en dos partes: una primera de carácter informativo general y una segunda en el aula donde ha empezado la ESO el alumno para conocer más de cerca el programa del curso y el lugar donde se desarrollará. Permite solucionar dudas y minimizar las preocupaciones que tienen algunas familias. 
- Tutor/a y alumno/a se entrevistan con la familia: El alumnado de ESO prepara la entrevista con su tutor o tutora. Una parte significativa de la entrevista la lleva el alumno que explica su evolución, sus dificultades y las estrategias y recursos necesarios para superarlas y los compromisos que todos adoptan en dicho proceso. Esta práctica supone situar al alumno realmente en el centro de su proyecto formativo y darle el protagonismo que, con el apoyo necesario, merece.

\section{CONCLUSIONES Y PROPUESTAS}

Tal y como figuraban en el informe de la investigación desarrollada, se propusieron 10 conclusiones que se resumen a continuación por su vigencia e interés.

1. Los IE que integran la educación infantil, primaria y secundaria en un mismo centro presentan una estructura equivalente a la de los centros educativos nórdicos en el modelo de sistemas integrados. En Catalunya, a partir de la Ley de Educación de Catalunya (LEC) se ha recuperado esta modalidad de centros que desde entonces ha ido creciendo lentamente al ser una iniciativa de éxito para contextos territoriales y socioeducativos muy diversos.

2. Las visiones que el estudio identificó fueron muy positivas en todas las dimensiones estudiadas. Los colectivos consultados (directivos, docentes, tutores y familias) mostraron elevados niveles de satisfacción y valoraciones positivas si bien los inicios de dichos centros no siempre fueron fáciles debido a diversas circunstancias. El hecho de tratarse de proyectos fuertes y de las dinámicas propias de cada IE hizo que las resistencias y las dificultades se fueran transformando en procesos colaborativos y en visiones positivas.

3. Las iniciativas territoriales estudiadas (de coordinación de centros de primaria y secundaria ordinarios) podrían calificarse de muy valiosas al permitir articular y coordinar acciones en el territorio articulando y vinculando centros de primaria y secundaria. Las formas de liderazgos fueron múltiples e implicaron a diversos agentes de los centros o de los servicios educativos que de manera esencialmente colaborativa hicieron posible el conjunto de experiencias que se reseñaron.

4. El estudio puso de manifiesto de manera explícita las dificultades de coordinación en los ámbitos más difíciles, el currículum, especialmente la coordinación de contenidos, metodologías y de sistemas de evaluación, así como la participación de las familias en el centro. También se pudo constatar el camino que queda por recorrer en relación a las relaciones del centro con su entorno.

5. El estudio permitió también perfilar las visiones que los distintos colectivos tienen cuando los centros son segregados en relación a las visiones de los colectivos de los IE. Si bien no se trató de una muestra significativa, sino que se estratificó intencionalmente en función de su proximidad y homologación socioeconómica, se pudo identificar claramente que las visiones de las personas vinculadas a los IE fueron claramente más positivas que cualquier otra visión de cualquier otro colectivo en todas las dimensiones consideradas. 
6. Los docentes de los institutos encuestados mostraron resultados cercanos a los de los IE si bien las familias del alumnado de estos centros mostraron una visión menos optimista, quizás porque en secundaria las familias y los centros disminuyen el nivel de interacción y el vínculo que tenían en infantil y primaria. En el caso de las escuelas, el colectivo más crítico son los docentes y las familias se muestran más optimistas. Probablemente los docentes estén especialmente sensibilizados con los itinerarios posteriores y muestren su preocupación por cuestiones como el retorno de información, que no siempre se produce. Si se atiende a los perfiles profesionales, los equipos directivos muestran una visión más optimista, seguidos de los responsables de coordinación mientras que los tutores de sexto y primero son los más críticos quizás porqué son los que afrontan directamente las responsabilidades de tutorización, coordinación e información.

7. Existen algunas dificultades en los IE que generan malestar entre sus profesionales, generalmente relacionadas con cuestiones administrativas, de cultura profesional de los dos cuerpos docentes o aquellas derivadas del reajuste de recursos asignados en la nueva estructura integrada, añadidas a las propias derivadas de la puesta en marcha de un nuevo centro, un nuevo proyecto, en ocasiones resultado de fusionar dos centros y en otros de transformar centros con alta complejidad. Debido a la corta historia de dichos centros y a las dinámicas identificadas, probablemente se puede pensar que en un tiempo razonable se podrán ir solucionando. El impulso de nuevos centros de estas características en estos últimos años parece confirmarlo.

8. Las entrevistas mantenidas con los equipos de dirección y coordinación de algunos IE y de algunos centros de primaria y de secundaria en los mismos territorios, sirvieron para documentar experiencias que pueden considerarse prácticas de referencia. Todas ellas muestran posibilidades de coordinar las dos etapas a través del trabajo compartido del profesorado y también del resto de docentes y la implicación del alumnado y las familias. Cabe señalar que todas las experiencias pueden ser útiles tanto para IE como para centros de primaria y de secundaria independientes que pueden tomarlas como inspiración para desarrollar sus propias prácticas. Por ello las descripciones fueron breves porqué sólo se trataba de sugerir posibilidades de acción y no descripciones de prácticas de referencia concretas que respondían a contextos concretos.

9. Resulta relevante destacar la participación del alumnado, además de la del profesorado, de las dos etapas, en buena parte de las prácticas referenciadas. Esta implicación del alumnado de las dos etapas educativas genera vínculos intergeneracionales que minimizan el impacto de las transiciones y traspasos y hacen fluidos los cambios generando sentimientos de pertenencia y de comunidad.

10. Finalmente, se constata que las familias siguen teniendo, salvo algunas excepciones, un papel de receptoras de información con escasa participación en el centro. Pocas prácticas identificadas van más allá de considerar a las familias como audiencia. El reto es pues conseguir mayor implicación y una comunicación más efectiva y bidireccional. 


\section{BIBLIOGRAFÍA}

Anderson, L., Jacobs, J., Schramm, S. y Splitgerber, F. (2000). School transitions: beginning of the end or a new beginning? International Journal of Educational Research, 33(4), 325-339). DOI: https://doi.org/10.1016/S0883-0355(00)00020-3

Dupriez, V., Dumay, X. y Vause, A. (2008). How Do School Systems Manage Pupils' Heterogeneity? Comparative Education Review, 52(2), 245-273. DOI: https://doi.org/10.1086/528764

EURYDICE (2005). Glosario Europeo sobre Educación. Instituciones educativas Vol. 2, 2a edición. Bruselas: Eurydice. Unité Européene. Disponible en: http://www.mec.es/cide/eurydice/index.htm [consulta: 3-9-2011].

EURYDICE (2011a). Cifras clave de la educación en Europa 2009. Bruselas: Eurydice. Unité Européene.

EURYDICE (2011b). Estructuras de los sistemas educativos de formación en Europa. España 2009/2010. Bruselas: Eurydice. Unité Européene.

EURYDICE (2011c). Fiche Nationale de synthèse des systèmes d'enseignement en Europe et des réformes en tours. France 2010. Bruselas: Eurydice. Unité Européene.

EURYDICE (2011d). National system overviews on education Systems in Europe and ongoing reforms. Germany 2010. Bruselas: Eurydice. Unité Européene.

EURYDICE (2011e). National system overviews on education Systems in Europe and ongoing reforms. Finland 2010. Bruselas: Eurydice. Unité Européene.

EURYDICE (2011f). National system overviews on education Systems in Europe and ongoing reforms. Norway 2010. Bruselas: Eurydice. Unité Européene.

EURYDICE (2011g). National system overviews on education Systems in Europe and ongoing reforms. Sweden 2010. Bruselas: Eurydice. Unité Européene.

EURYDICE (2011h). Resumen descriptivo de los sistemas educativos europeos y las reformas en curso. España 2010. Bruselas: Eurydice. Unité Européene.

EURYDICE (2011i). Structures of Education and Training Systems in Europe. Germany 2009/2010. Bruselas: Eurydice. Unité Européene.

EURYDICE (2011j). Structures of Education and Training Systems in Europe. Finland 2009/2010. Bruselas: Eurydice. Unité Européene.

EURYDICE (2011k). Structures des systèmes d'enseignement et de formation en Europe. France 2009/2010. Bruselas: Eurydice. Unité Européene.

EURYDICE (20111). Structures of Education and Training Systems in Europe. Norway 2009/2010. Bruselas: Eurydice. Unité Européene.

EURYDICE (2011m). Structures of Education and Training Systems in Europe. Sweden 2009/2010. Bruselas: Eurydice. Unité Européene.

EURYDICE (2011n). The structure of the European education Systems 2011 / 2012. Schematic diagrams. Bruselas, Eurydice. Unité Européene. Disponible en: http://eacea.ec.europa.eu/ education/eurydice/tools_en.php\#diagrams [Consulta: 8-9-2011].

EURYDICE (2011o). Grade Retention during Compulsory Education in Europe: Regulations and Statistics. Bruselas: Eurydice. Unité Européene. Disponible en: http://eacea.ec.europa.eu/ education/eurydice/thematic_studies_en.php [Consulta: 20-11-2011]. 
EURYPEDIA (2011a). Guidance and Counselling in Early Childhood and School Education. Finland. European Commission, EACEA, Eurydice. Disponible en: https:/webgate.ec.europa. eu/fpfis/mwikis/eurydice/index.php/Finland:Guidance_and_Counselling_in_Early_Childhood_and_School_Education [Consulta: 5-12-2011].

EURYPEDIA (2011b). Guidance and Counselling in Early Childhood and School Education. France. European commission, EACEA, Eurydice. Disponible en: https://webgate.ec.europa.eu/ fpfis/mwikis/eurydice/index.php/France:Guidance_and_Counselling_in_Early_Childhood_ and_School_Education [Consulta: 8-12-2011].

EURYPEDIA (2011b). Guidance and Counselling in Early Childhood and School Education. Germany. European commission, EACEA, Eurydice. Disponible en: https://webgate.ec.europa. eu/fpfis/mwikis/eurydice/index.php/Germany:Organisation_of_General_Lower_Secondary_EducationAdmission_requirements_and_choice_of_school [Consulta: 5-12- 2011].

EURYPEDIA (2011d). Guidance and Counselling in Early Childhood and School. Education. Norway. European commission, EACEA, Eurydice. Disponible en: https://webgate. ec.europa.eu/fpfis/mwikis/eurydice/index.php/Norway:Guidance_and_Counselling_in_ Early_Childhood_and_School_Education [Consulta: 5-12-2011].

EURYPEDIA (2011e). Guidance and Counselling in Early Childhood and School Education Spain. European commission, EACEA, Eurydice. Disponible en: https://webgate.ec.europa.eu/ fpfis/mwikis/eurydice/index.php/Spain:Guidance_and_Counselling_in_Early_Childhood_ and_School_Education [consulta: 5-12-2011].

EURYPEDIA(2011f). Guidance and Counselling in Early Childhood and School Education. Sweden. European commission, EACEA, Eurydice. Disponible en: https://webgate.ec.europa.eu/fpfis/mwikis/eurydice/index.php/Norway:Guidance_and_Counselling_in_Early_Childhood_ and_School_Education [consulta: 10-12-2011].

Galton, M. y Morrison, I. (2000). Concluding comments. Transfer and transition: the next steps. International Journal of Educational Research, 33(4), 443-449. DOI: https://doi.org/10.1016/ S0883-0355(00)00027-6

Galton, M., Morrison, I y Pell, T. (2000). Transfer and transition in English schools: reviewing the evidence. International Journal of Educational Research, 33(4), 341-363. DOI: https://doi. org/10.1016/S0883-0355(00)00021-5

Gimeno Sacristán, J. (1996). La Transición a la Educación Secundaria. Discontinuidades en las Culturas Escolares. Madrid: Morata.

Gradaïlla, A., Querol, C. y Bundó, M. (2011). El pas de l'etapa d'educació primària a educació secundària. Escola Catalana, 470, 21-16. Disponible en: http://www.omnium.cat/ca/escolacatalana/transicions-entre-etapes_574.html[Consulta: 5-12-2011].

Gratacós, P y Ugidos, P. (2011). Diversitat cultural i exclusió escolar. Dinàmiques educatives, relacions interpersonals i actituds del professorat. Barcelona: Fundació Jaume Bofill. Disponible en: www.fbofill.cat/d.php?docID=545\&t=1 [Consulta: 12 -12-2011].

Hargreaves, A. (1994). Teacher's work and culture in the postmodern age. Londres, Cassell.

Hargreaves, A. y Galton, M. (2002). Transfer from the primary classroom. 20 years on. Nova York: Routledge. 
Martínez, M. y Pinya, C. (2012). Els instituts escola: aspectes curriculars, organitzatius $i$ d'orientació. Documents núm. 21. Barcelona: Departament d'Ensenyament - Consell Superior d'avaluació del Sistema Educatiu - Institut de Ciències de l'Educació - UAB.

OECD (1999). Classifying Educational Programmes. Manualfor ISCED-97 Implementation in OECD Countries. París: OECD. Disponible en: http://www.oecd.org/dataoecd/41/42/1841854.pdf [Consulta: 1-10-2011].

OECD (2010). Education at a Glance OECD Indicators 2010 Annex 3: Sources, methods and technical notes. Chapter D: The learning environment and organisation of schools. París: OECD. DOI: https://doi.org/10.1787/eag-2010-en

OECD (2011a). Education at a Glance OECD Indicators 2011. París: OECD. DOI: http://dx.doi. org/10.1787/eag-2011-en

OECD (2011b). Improving lower secondary schools in Norway. Reviews of National Policies for Education. París: OECD. DOI: http://dx.doi.org/10.1787/9789264114579-en

Palacios, L. (1988). Instituto-Escuela. Historia de una renovación educativa. Madrid, Ministerio de Educación y Ciencia.

Pietarinen, J. (2000). Transfer to and study at secondary school in Finnish school culture: developing schools on the basis of pupils' experiences, International Journal of Educational Research, 33(4), 383-400. DOI: https://doi.org/10.1016/S0883-0355(00)00024-0

Ruiz Guevara L. S. y otros (2010). Transición a la secundaria: los temores y preocupaciones que experimentan los estudiantes de primaria. Revista Iberoamericana de Educación, 52(3). Disponible en: http://www.rieoei.org/deloslectores/3253Ruiz.pdf

Scott, L. S., Rock, D. A., Pollack, J. e Ingels, S. J. (1985). Two years later: Cognitive gains and school transitions of NELS: 88 eighth graders. Washington, DC: National Center for Educational Statistics.

UNESCO (2006). World data on Education. Disponible en: http:/www.ibe.unesco.org/Countries/ WDE/2006/index.html [Consulta: 1-10-2011].

Vargas J. D. y Villares, L. (2010). La coordinació entre primària i secundària des d'un enfocament orientador. Guix, 370, 57-63.

Villares, L. y Vargas, J. D. (2006). Com organitzar un seminari intercanvi d'experiències primàriasecundària: la transició com a procés. Perspectiva Escolar, 303, 66-70.

Ward, R. (2000). Transfer from middle to secondary school: A New Zealand study, International Journal of Educational Research, 33(4), 365-374. DOI: https://doi.org/10.1016/S08830355(00)00022-7 